\title{
Predictors of adverse birth outcomes among pregnant adolescents in Ashanti Region, Ghana
}

\author{
Reginald Adjetey Annan ${ }^{1 *}$, Linda Afriyie Gyimah ${ }^{1}$ (D), Charles Apprey ${ }^{1}$, Odeafo Asamoah-Boakye ${ }^{1}$, \\ Linda Nana Esi Aduku ${ }^{1}$, Wisdom Azanu ${ }^{2}$, Herman E. Luterodt ${ }^{3}$ and Anthony K. Edusei ${ }^{4}$ \\ ${ }^{1}$ Human Nutrition and Dietetics Unit, Department of Biochemistry and Biotechnology, Faculty of Biosciences, College of Science, Kwame Nkrumah \\ University of Science and Technology, Kumasi, Ghana \\ ${ }^{2}$ Department of Obstetrics and Gynecology, University of Allied Health Sciences, Ho, Ghana \\ ${ }^{3}$ Department of Food Science and Technology, Kwame Nkrumah University of Science and Technology, Kumasi, Ghana \\ ${ }^{4}$ Department of Community Health, School of Public Health, Kwame Nkrumah University of Science and Technology, Kumasi, Ghana
}

(Received 31 December 2020 - Final revision received 19 July 2021 - Accepted 22 July 2021

Journal of Nutritional Science (2021), vol. 10, e67, page 1 of 11

doi: $10.1017 /$ jns. 2021.58

Abstract

Adolescent pregnancy is associated with adverse birth outcomes. However, the determinants of these outcomes are understudied. The present study sought to identify the predictors of adverse birth outcomes among pregnant adolescents in Ghana. In this prospective health centre-based study, 416 pregnant adolescents, aged 13-19 years old, were followed, and 270 birth outcomes were evaluated. We collected data on socio-demographic variables, eating behaviour, household hunger scale (HHS), lived poverty index (LPI) and compliance to antenatal interventions. The prevalence of low birth weight (LBW) and preterm births (PTB) were 15.2 and $12.5 \%$, respectively. Pregnant adolescents with no formal education (AOR 9.0; P=0.004; $95 \%$ CI 2.1, 39.8), those who experienced illness (AOR 3.0; $P=0 \cdot 011 ; 95 \% \mathrm{CI} 1 \cdot 3,7 \cdot 0)$, those who experienced hunger (OR 2.9; $P=0 \cdot 010 ; 95 \%$ CI 1.3, 6.5) and those with high LPI (OR 2.5; $P=0.014 ; 95 \%$ CI $1 \cdot 2,5 \cdot 3$ ) presented increased odds of delivering preterm babies compared with those who have had secondary education, did not experience any illness, were not hungry or having low LPI, respectively. Pregnant adolescents who used insecticide-treated net (ITN) (AOR $0 \cdot 4$; $P=0.013 ; 95 \% \mathrm{CI} 0 \cdot 2,0 \cdot 9$ ) presented reduced odds LBW children; while those who experienced illness (AOR $2 \cdot 7 ; P=0 \cdot 020 ; 95 \% \mathrm{CI} 1 \cdot 2,6 \cdot 0$ ), poorer pregnant adolescents (OR 2.5; $P=0 \cdot 014 ; 95 \%$ CI 1.1, 4.8) and those who experienced hunger (AOR 3.0; $P=0 \cdot 028 ; 95 \%$ CI 1.1, 8.1) presented increased odds of LBW children compared with those who used ITN, were not ill, were not poor or did not experience hunger. Adverse birth outcomes were associated with ANC compliance and socioeconomic factors of the pregnant adolescents. Hence, strengthening antenatal uptake and compliance by pregnant adolescents, promoting their livelihood and socioeconomic status, and interventions to prevent teenage pregnancies are strongly recommended.

Key words: Adolescent pregnancy: Birth outcomes: Household hunger scale: Lived poverty index: Low birth weight: Preterm

\section{Introduction}

Events that occur during the first $1000 \mathrm{~d}$ (from conception through to 2 years) in a child's life, including birth outcomes, are significant. They contribute to the commencement of proper development and impact future health ${ }^{(1)}$. Birth outcomes, including birth weight, gestational age, abortion, neonatal mortality and stillbirths, are a measure of babies' health at birth. Of these, the two most studied indicators are birth weight and gestational age, yet their causes are not fully understood $^{(2)}$. Worldwide, low birth weight (LBW) is estimated at $15-20 \%$ births $^{(3)}$, and 1.1 million babies die as a result of preterm birth complications annually ${ }^{(4)}$. The WHO estimates that more than 9 million infants were dying within their first birthday or even before birth every year, especially in developing countries $^{(5)}$. The inequality in birth outcomes between lowand high-income countries is attributed to poor antenatal

* Corresponding author: Reginald Adjetey Annan, email reggie@imtf.org

(C) The Author(s), 2021. Published by Cambridge University Press on behalf of The Nutrition Society. This is an Open Access article, distributed under the terms of the Creative Commons Attribution licence (http://creativecommons.org/licenses/by/4.0/), which permits unrestricted re-use, distribution, and reproduction in any medium, provided the original work is properly cited. 
care (ANC) received by pregnant women in low-income countries ${ }^{(6)}$. Birth outcomes are generally related to social, economic, biological and environmental factors ${ }^{(7-10)}$.

Maternal nutrition practices, child growth period and the intrauterine environment during pregnancy may also affect birth outcomes ${ }^{(11-14)}$. A study conducted in Ghana on birth weight concluded that mothers with adequate dietary practices were less likely to have babies with $\mathrm{LBW}^{(15)}$. Poor maternal dietary habits have been related to rural living, food insecurity and poverty ${ }^{(16)}$. Moreover, unfavourable birth outcomes have been related to low socioeconomic status such as low education level, low income, poor access to health facilities and poor housing conditions ${ }^{(17,18)}$. Adverse birth outcomes have also been related to maternal morbidity and insufficient mater-

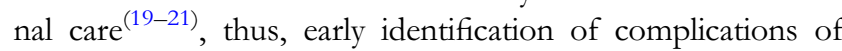
pregnancy during antenatal is vital.

ANC is the basic component of maternal care on which the life of mothers and babies depend ${ }^{(22)}$. According to the $\mathrm{WHO}^{(23)}$, ANC is part of the three most important care given to pregnant women and a crucial indicator of reducing the global maternal mortality ratio as specified in target 3 of the Sustainable Development Goal (SDG). Accessing ANC services have been directly linked to better birth outcomes and a decrease in infant mortality and malnutrition among low-income and middle-income countries ${ }^{(24)}$. ANC services are also related to socio-demographic or reproductive characteristics, and consequently birth outcomes ${ }^{(25-28)}$. The Government of Ghana endorsed the Focused Antenatal Care (FANC) strategy of the World Health Organization (WHO) in 2002 to address the significantly higher maternal mortality rate and to increase access, quality and consistency of ANC for pregnant women. ANC is a focal point for pregnant women and their fetuses for receiving a wide variety of preventive and healthcare activities, treatment, monitoring during pregnancy up to delivery ${ }^{(29)}$. There is late initiation of ANC attendance among pregnant adolescents as they are more likely to hide their pregnancy due to doubt and naivety, lack of finance and stigmatisation ${ }^{(30)}$. Studies among pregnant adolescents have reported a high risk for adverse birth outcomes, such as preterm, fetal death or $\mathrm{LBW}^{(31-35)}$. Furthermore, findings have reported an even higher risk for unfavourable birth outcomes in much younger adolescents $^{(36,37)}$. The $\mathrm{WHO}^{(38)}$ reported an estimation of $12 \cdot 8$ million births (44 births per 1000 adolescent girls) of teenage girls between 15 and 19 years in 2018, with the highest occurrence in low-income countries (8 times higher), compared with high-income countries.

A maternal health survey conducted in 2017 in Ghana identified $12 \%$ miscarried pregnancies, $2 \%$ stillbirths and $10 \%$ induced abortions among women aged 15-49 years ${ }^{(39)}$. Among these women of reproductive age, the prevalence of adolescent pregnancy was $14.2 \%$, with stillbirths, miscarriages and induced abortion recording $1 \cdot 3,6 \cdot 8$ and $18.8 \%$, respectively. Live births among this group were the least $(73 \cdot 1 \%)$ as compared with the adult group $(77.4 \%)$, who were between 20 and 34 years $^{(39)}$. A study conducted in Kumasi, Ghana, recorded a prevalence of $19 \%$ adverse birth outcomes ${ }^{(40)}$ while an earlier one reported a $44.6 \%$ prevalence $^{(41)}$ among pregnant women. Among adolescents in Kumasi, the second-largest city in Ghana, $23 \%$ had $\mathrm{LBW}^{(42)}$.

The seventh target of the SDG 3 seeks to ensure universal access to sexual and reproductive healthcare services. To achieve this goal, the Ministry of Gender, Children and Social Protection in Ghana developed a five-year strategic plan (2018-22). The plan seeks to reach adolescents with the correct information and insight and to provide them with training and services to protect them from early and unintended pregnancies ${ }^{(43)}$. Despite this, adolescent pregnancies seem to be on the rise. To prepare them for desirable birth outcomes, a better understanding of the determinants of birth outcomes in this group is required. WHO's global target for 2025 is to reduce LBW by $30 \%$, that is, a $3 \%$ annually reduction. It is expedient that we identify the factors which lead to these adverse birth outcomes among this age bracket. The present study was, therefore, conducted to explore the predictors of adverse birth outcomes among pregnant adolescents in Ashanti Region, Ghana.

\section{Methods}

\section{Study design}

In this prospective health centre-based study, 416 pregnant adolescents (aged 13-19 years old) with a maximum of 32 gestational weeks were recruited from May to December 2018 and followed until they gave birth between January and July 2019.

\section{Study area and population}

Ashanti Region, the most populated region in Ghana, makes up $19 \%$ of Ghana's total population, which is about 5792187 people ${ }^{(44)}$. The prevalence of adolescent pregnancy in the region is $12.2 \%$, according to a recent survey conducted in $2017^{(39)}$. We recruited participants from health centres serving communities in three urban districts (Kumasi Metropolis, Asante Akim Central Municipal and Ejisu Juaben Municipal) and five rural districts (Bosomtwi, Asante Akim South and North, and Ahafo Ano North and South) in Ashanti Region, Ghana. We recurited participants at selected Community-based Health Planning Services (CHPS) compounds, health centres and hospitals in the selected districts ${ }^{(44)}$.

\section{Sample size}

The sample size was statistically calculated using the sample size-based proportion formula by ${ }^{(45)}: n=2(Z \alpha / 2+Z \beta) 2$ $p(1-p) /(P 1-P 2)^{2}$. Where $n$ is the sample size, $Z \alpha / 2=$ 1.96 at type 1 error of $5 \%, Z \beta=0.84$ at $80 \%$ power, $P 1$ is the LBW in pregnant adolescents with adequate nutritional status, $P 2$ is the LBW in pregnant adolescents with poor nutritional status, $P 1-P 2$ is the difference in the prevalence of LBW between pregnant adolescents with adequate nutritional status at birth and those with inadequate nutritional status, and $P$ is pooled prevalence $=(P 1+P 2) / 2$. Based on a previous pilot study by Ayensu et al. ${ }^{(42)}$, which reported $23 \%$ LBW prevalence, we proposed that LBW in pregnant adolescents with adequate nutritional status would be $11.4 \%$, while 
those with poor nutritional status would remain $23 \%$. Using the above descriptives, the sample size $n=2(1.96+0.84)^{2} \times$ $0 \cdot 1735(1-0 \cdot 233) /(0 \cdot 114-0 \cdot 233)^{2}, n=2 \cdot 09 / 0 \cdot 01, n=209$ was calculated, which implied we needed to recruit 209 participants in each arm of the study (half in the poorly nourished group and a half in the well-nourished group) making 418 participants to show a significant association between poor nutrition and LBW. We added $10 \%$ attrition to give 460 participants who were needed.

However, 416 pregnant adolescents were recruited on a first-come, first-served basis for the baseline study. Trained enumerators visited hospitals/health centres selected for the study on antenatal clinic days for pregnant adolescents. All pregnant adolescents within the inclusion criteria who gave written consents were written. In the rural areas, announcements were made at the community information centres to invite participants to the health centres on specific days. These announcements were necessary as some pregnant adolescents refused to visit the health centres for ANC to fear being stigmatised. We trained enumerators in a two-day workshop on each data collection tool used. We obtained birth outcome data from 270 (birth weight) and 303 (gestational age) participants out of the 416 recruited during the baseline study as some participants were lost to follow-up.

\section{Ethics}

Ethical approval for the study was obtained from the Committee on Human Research Publication and Ethics (CHRPE) of the Kwame Nkrumah University of Science and Technology, KNUST, (Kumasi, Ghana) (CHPRE/AP/ 236/18). Study protocols/aims were first explained to all participants in their local language. Written and signed informed consent was obtained from all participants by following CHRPE regulations before recruiting for the study.

\section{Data collection tools}

A structured questionnaire was used to collect data on the socio-demographic variables, dietary diversity, eating behaviours (pica, food aversion, food craving), food deprivation (household hunger scale - HHS), availability of necessities (lived poverty index - LPI), antenatal interventions compliance (nutrition education, micronutrient supplementation, tetanus injection, malaria tablet intake, use of the ITN, daily intake of micronutrient), maternal factors (maternal morbidity and mode of delivery) and birth outcomes (birth weight and gestational age) of the pregnant adolescents. Data on age and parity were verified from their National Health Insurance Identification cards and maternal health record book. The questionnaire was pretested at selected health centres and validated to ensure appropriate responses from participants. Birth weight and gestational age were the dependent variables.

\section{Assessment of dietary diversity}

A previous day's 24-h dietary recall method was used to assess dietary diversity among the participants. The FAO's Minimum Dietary Diversity for Women (MDD-W), was used to determine maternal dietary diversity using ten food groups. The MDD-W score obtained was then used to identify whether maternal dietary diversity was adequate (5-10 food groups) or not (0-4 food groups).

\section{Assessment of eating behaviour}

Questions on whether or not the participants were practising food cravings, pica and food aversions were asked. Each question had a yes/no optional response.

\section{Assessment of HHS and LPI}

We collected data on food availability and deprivation over the past month using standardised close-ended questionnaires ${ }^{(46)}$ to assess hunger (HHS) among participants. The responses to these questions were then coded and scored. Scores between $<2$ meant little or no hunger, 2-3 meant moderate hunger and 4-6 meant severe hunger. LPI was assessed by asking questions on the availability of food, water, cash income, medical care and cooking fuel over the past year. The responses were coded and scored. A score of 0 meant no lived poverty, while the scores of 1-4 meant the absence of one or more necessities $^{(47)}$. The averages of the scores were then categorised into low $(0-1 \cdot 0)$, moderate $(1 \cdot 01-1 \cdot 5)$ and high $(>1 \cdot 5)$ LPI.

\section{Assessment of antenatal interventions}

A structured questionnaire was administered to participants to identify their compliance with the antenatal interventions given at their various health facilities. Participants were asked if they received nutrition education, tetanus injection, micronutrient supplements, malaria tablet, ITN, consumed daily micronutrient supplement, and whether they used the ITN given.

\section{Assessment of birth outcomes and maternal factors}

Follow-up data on several birth outcomes, including birth weight and gestational age at birth were obtained from their maternal health record books and were categorised as normal or adverse. LBW was defined as a birth weight of less than $2 \cdot 5$ $\mathrm{kg}^{(28)}$. Preterm birth was defined as delivery less than $37 \mathrm{com}-$ pleted weeks of gestation ${ }^{(48)}$. Questions on mode of delivery (vaginal delivery, caesarean section) and morbidity during pregnancy were asked.

\section{Data analysis}

Microsoft Excel was used to clean the entered data, and Statistical Package for Social Sciences version 25 (SPSS IBM Inc., Chicago, USA) was used to analyse them. We performed $\chi^{2}$ (Fischer's exact test) and cross-tabulation to compare frequencies of all variables used (antenatal interventions and compliance, maternal factors and birth outcomes) among community types. Frequencies of birth weight and gestational age were compared among socio-demographic variables, dietary diversity, eating behaviours, LPI, HHS, antenatal interventions compliance and maternal factors using Fischer's exact test. We used logistic regression to identify predictors of 
Table 1. Differences in socio-demographic factors and birth outcomes (birth weight and gestational age)

\begin{tabular}{|c|c|c|c|c|c|c|c|}
\hline \multirow[b]{2}{*}{ Variables } & \multirow{2}{*}{$\begin{array}{l}\text { Total } \\
n(\%)\end{array}$} & \multicolumn{2}{|c|}{ Birth weight } & \multirow[b]{2}{*}{$P$ value } & \multicolumn{2}{|c|}{ Gestational age } & \multirow[b]{2}{*}{$P$ value } \\
\hline & & LBW & NBW & & Preterm & Term & \\
\hline Age & & & & $0.360^{b}$ & & & $0.487^{b}$ \\
\hline $13-15$ years & $32(7 \cdot 7)$ & $4(22 \cdot 2)$ & $16(77 \cdot 8)$ & & $1(5 \cdot 0)$ & $19(95.0)$ & \\
\hline $16-19$ years & $384(92 \cdot 3)$ & $37(14.8)$ & $213(85 \cdot 2)$ & & $37(13 \cdot 1)$ & $246(86.9)$ & \\
\hline Marital Status & & & & $0.326^{b}$ & & & $0.428^{b}$ \\
\hline Single & $316(76 \cdot 0)$ & $28(13.8)$ & $175(86 \cdot 2)$ & & $26(11.6)$ & $199(88.4)$ & \\
\hline Married & $100(24.0)$ & $13(19.4)$ & $54(80 \cdot 6)$ & & $12(15.4)$ & $66(84.6)$ & \\
\hline Occupation & & & & $0.257^{b}$ & & & $0.176^{b}$ \\
\hline Unemployed & $298(71 \cdot 6)$ & $33(15 \cdot 6)$ & $163(84.4)$ & & $24(10.9)$ & $196(86 \cdot 4)$ & \\
\hline Employed & $118(28.4)$ & $8(16.9)$ & $66(83.1)$ & & $14(16.9)$ & $69(83.1)$ & \\
\hline Parity & & & & $0.845^{\mathrm{b}}$ & & & $1.000^{\mathrm{b}}$ \\
\hline 1 & $316(76 \cdot 0)$ & $30(14 \cdot 9)$ & $172(85 \cdot 1)$ & & $29(12 \cdot 8)$ & $198(87 \cdot 2)$ & \\
\hline$>1$ & $100(24.0)$ & $11(16 \cdot 2)$ & $57(83 \cdot 8)$ & & $9(11 \cdot 8)$ & 67 (88.2) & \\
\hline Education & & & & $0.428^{a}$ & & & $0.006^{a}$ \\
\hline None & $19(4 \cdot 6)$ & $2(16 \cdot 7)$ & $10(83.3)$ & & $6(40 \cdot 0)$ & $9(60)$ & \\
\hline Primary & $56(13.5)$ & $3(8 \cdot 8)$ & $31(91 \cdot 2)$ & & $2(5 \cdot 1)$ & 37 (94.9) & \\
\hline JHS & $255(61 \cdot 3)$ & $25(14.5)$ & $148(85.5)$ & & $23(12 \cdot 2)$ & $165(87.8)$ & \\
\hline SHS & $86(20 \cdot 7)$ & $11(21 \cdot 6)$ & $40(78.4)$ & & $7(11.5)$ & $54(88.5)$ & \\
\hline Income & & & & $0.517^{\mathrm{a}}$ & & & $0.094^{a}$ \\
\hline No Income & $310(74.5)$ & $28(13 \cdot 6)$ & $178(86.4)$ & & $23(10.0)$ & $206(90 \cdot 0)$ & \\
\hline Below 100 & $41(9 \cdot 9)$ & $4(17.4)$ & $19(82 \cdot 6)$ & & $7(23 \cdot 3)$ & $26(76 \cdot 7)$ & \\
\hline Between 100-500 & $61(14 \cdot 7)$ & $8(21 \cdot 1)$ & $30(78.9)$ & & $8(19 \cdot 0)$ & $34(81.0)$ & \\
\hline Between 500-1000 & $4(1 \cdot 0)$ & $1(33 \cdot 3)$ & $2(66 \cdot 7)$ & & 0 & $2(100)$ & \\
\hline
\end{tabular}

HHS, Household Hunger Scale; LPI, Lived Poverty Index.

Bold values are significant at $P<0.05$.

${ }^{\mathrm{a}} \chi^{2}$.

${ }^{\mathrm{b}}$ Fischer's exact test.

birth outcomes. We presented the data as frequency $(\%)$ and $P$ values $<0.05$ were considered significant.

\section{Results}

\section{Impact of socio-demographic factors on birth outcomes}

The distribution of socio-demographic characteristics of pregnant adolescents and among community types used for the present study has been presented in another study ${ }^{(16)}$. Effects of socio-demographic status on birth outcomes are presented in Table 1. Among all the factors used, the level of education affected the gestational age at birth significantly $(P=0 \cdot 006)$. Generally, preterm births (PTBs) decreased with increasing education level (none $=40 \%$; SHS $=11.5 \%$ ). PTBs showed slightly different trends by socio-demographic status. Older adolescents (16-19 years) recorded more PTBs $(13.1 \%)$ than adolescents in the younger bracket (5\%). Similarly, more PTB's were recorded among adolescents who were married $(19.4 \%)$ and employed $(16.9 \%)$ than among those who were single $(13.8 \%)$ and unemployed $(10.9 \%)$. LBWs were proportionally higher among adolescents who were younger than their older counterparts $(22.2 \% v .14 .8 \%)$, married than single $(19.4 \% \quad v \cdot 13.8 \%)$, employed than unemployed $(16.9 \% v .15 .6 \%)$ and among those with highincome range than those without income $(33.3 \% v .13 .6 \%)$.

\section{ANC service delivery and uptake, maternal factors and birth outcomes}

Table 2 presents the frequencies of antenatal interventions, maternal factors and participants' birth outcomes in the present study. Levels of participants who received nutrition education $(P=0.022)$, tetanus injection $(P=0.021)$, used insecticide-treated nets $(P=0.026)$ and mode of delivery $(P=0.009)$ varied significantly between rural and urban study participants. Generally, about half of pregnant adolescents $(49.5 \%)$ received nutrition education during antenatal services. Nearly $12 \%$ more of pregnant adolescents from urban areas received nutrition education than their rural counterparts $(P=0.022)$. About $60.6 \%$ received micronutrient supplements while only $46 \%$ were taking their supplements daily. Four ${ }^{(4)}$ out of ten pregnant girls had not received tetanus injection. However, a more significant proportion of urban girls $(P=0.021)$ had received tetanus injection. About four to five out of ten had not received malaria tablets or insecticide-treated net (ITN). About $17 \%$ of pregnant girls who had received the ITN were not using them. The use of ITN among rural girls was significantly higher $(P=0.026)$ than those in urban areas. Though not significant, LBW was higher among deliveries by rural girls $(18.8 \%)$ than girls $(13.4 \%)$. Also, preterm births proportion was higher among urban girls $(13.1 \%)$ than rural $(11.9 \%)$. About eight in ten pregnant girls delivered their babies using the vaginal method. Significantly, more rural girls $(P=0.009)$ than urban girls delivered through the vaginal method.

\section{Impact of antenatal service delivery and uptake, and maternal factors and birth outcomes}

Table 3 presents the effect of antenatal intervention uptake and maternal factors on birth outcomes. Adolescents who reported ill while pregnant showed a significantly larger 
Table 2. Antenatal care interventions and uptake, maternal factors and birth outcomes between rural and urban-dwelling pregnant adolescents

\begin{tabular}{|c|c|c|c|c|}
\hline Variable & $\begin{array}{c}\text { Total } n \\
(\%)\end{array}$ & $\begin{array}{c}\text { Rural } n \\
(\%)\end{array}$ & $\begin{array}{l}\text { Urban } n \\
(\%)\end{array}$ & $\begin{array}{c}P \\
\text { value }\end{array}$ \\
\hline Nutrition Education & & & & 0.022 \\
\hline Yes & $206(49.5)$ & $74(42 \cdot 8)$ & $132(54 \cdot 3)$ & \\
\hline No & $210(50.5)$ & $99(57 \cdot 2)$ & $111(45 \cdot 7)$ & \\
\hline $\begin{array}{l}\text { Micronutrient } \\
\text { Supplementation }\end{array}$ & & & & 0.186 \\
\hline Yes & $252(60 \cdot 6)$ & $98(56 \cdot 6)$ & $154(63.4)$ & \\
\hline No & $164(39.4)$ & $75(43.4)$ & $89(36 \cdot 6)$ & \\
\hline Tetanus Injection & & & & 0.021 \\
\hline Yes & $237(57)$ & $87(50 \cdot 3)$ & $150(61 \cdot 7)$ & \\
\hline No & $179(43)$ & $86(49 \cdot 7)$ & $93(38 \cdot 3)$ & \\
\hline Insecticide-Treated Net & & & & 0.108 \\
\hline Yes & $243(58.4)$ & $93(53.8)$ & $150(61 \cdot 7)$ & \\
\hline No & $173(41.6)$ & $80(46 \cdot 2)$ & $93(38.3)$ & \\
\hline Malaria Tablet Intake & & & & 0.686 \\
\hline Yes & $168(40.4)$ & $72(41 \cdot 6)$ & $96(39.5)$ & \\
\hline No & $248(59.6)$ & $101(58.4)$ & $147(60.5)$ & \\
\hline \multicolumn{5}{|l|}{$\begin{array}{l}\text { Daily Intake of } \\
\text { Supplements }\end{array}$} \\
\hline Yes & $190(45 \cdot 7)$ & $79(45 \cdot 7)$ & $111(45 \cdot 7)$ & \\
\hline No & $226(54 \cdot 3)$ & $94(54 \cdot 3)$ & $132(54 \cdot 3)$ & \\
\hline Use of ITN & & & & 0.026 \\
\hline Yes & $172(41 \cdot 3)$ & $83(48 \cdot 0)$ & $89(36 \cdot 6)$ & \\
\hline No & $244(58 \cdot 7)$ & $90(52 \cdot 0)$ & $154(63.4)$ & \\
\hline Birth weight & & & & 0.495 \\
\hline LBW $(<2.5 \mathrm{~kg})$ & $41(15 \cdot 2)$ & $20(17 \cdot 1)$ & $21(13 \cdot 7)$ & \\
\hline Normal ( $\geq 2.5 \mathrm{~kg}$ ) & $229(84 \cdot 8)$ & $97(82 \cdot 9)$ & $132(86 \cdot 3)$ & \\
\hline Gestational Age & & & & 0.862 \\
\hline Preterm (<37 weeks) & $38(12.5)$ & $17(11.9)$ & $21(13 \cdot 1)$ & \\
\hline Term ( $\geq 37$ weeks) & $265(87.5)$ & $126(88 \cdot 1)$ & $139(86 \cdot 9)$ & \\
\hline Maternal Morbidity & & & & 0.099 \\
\hline Yes & $55(17)$ & $29(21 \cdot 3)$ & $26(13.9)$ & \\
\hline No & $268(83)$ & $107(78 \cdot 7)$ & $161(86 \cdot 1)$ & \\
\hline Delivery Method & & & & 0.009 \\
\hline Vaginal & $285(88.2)$ & $142(93.4)$ & $143(83 \cdot 6)$ & \\
\hline Caesarean & $38(11.8)$ & $10(6 \cdot 6)$ & $28(16.4)$ & \\
\hline
\end{tabular}

LBW, Low Birth Weight.

Categorical data are presented as frequency (\%).

Bold values are significant at $P<0.05$.

proportion of LBWs $(27 \cdot 1 \%)$ than those who did not $(12 \cdot 6$ $\% ; P=0.024)$. Also, PTBs were over twice more among those reporting ill $(24.1 \%)$ than those who reported no illness $(10 \% ; P=0 \cdot 011)$. LBW was significantly lower among participants who used ITNs $(9.3 \% ; P=0.038)$ as compared with those who did not (19\%). Also, LBW was slightly lower among adolescents who received nutrition education but higher among those who took micronutrient supplements, and malaria tablets, tetanus injection, than their counterparts who did not though these did not reach a statistical significance. Though not significant, participants who delivered through caesarean section $(20.7 \%$ ) gave birth to a greater proportion of babies with LBW as compared with those who gave birth through the vaginal delivery method $(15 \cdot 8 \%)$.

Table 4 shows the effect of the HHS, LPI, eating behaviour and MDD on birth outcomes. Among these variables, HHS had a statistically significant effect on birth weights $(P=0.006)$ and PTB $(P=0.028)$, whereby adolescent girls who suffered hunger had a larger proportion of LBW (no hunger $=12 \%, \quad$ moderate $=35.5 \%$ and severe $=16.1 \%$; $P=0.006$ ) and PTB (no hunger $=10 \%$, moderate $=24.4 \%$ and severe $=13.2 \% ; P=0 \cdot 028)$. Likewise, poorer pregnant adolescent had significantly more LBW $(P=0.029)$ and PTB $(P=0 \cdot 006)$. Although not statistically significant, LBW was lower among those with adequate MDD (11.4\%) as compared with those with inadequate MDD (17.9\%), while PTB was rather lower among those with inadequate MDD $(11.6 \%)$ than those whose MDD were adequate $(13.7 \%)$. Similarly, those who practiced PICA had more LBWs $(16.2 \%)$ and less PTBs $(10.7 \%)$ than those who did not (LBW = $14 \cdot 6 \%, P=0 \cdot 057$; PTB $=13 \cdot 6 \%, P=0.590)$.

\section{Predictors of adverse birth outcomes}

Table 5 shows the findings of logistic regression on predictors of LBW. The use of ITN, LPI, HHS and maternal morbidity were significantly associated with delivering LBW babies. Pregnant adolescents who used ITN presented reduced odds (AOR $0 \cdot 4 ; P=0.013 ; 95 \%$ CI $0.2,0.9$ ) of giving birth to babies with LBW compared with those who did not use them. Those who were poorer, using LPI presented increased odds (OR 2.5; $P=0 \cdot 014 ; 95 \%$ CI 1.1, 4.8) of delivering LBW babies than those with low LPI. Also, pregnant adolescents who were moderately hungry had a higher risk of delivering LBW babies (AOR 3.0; $P=0 \cdot 028$; $95 \%$ CI 1.1, 8.1) compared with those who were not hungry. Maternal morbidity increased odds (AOR 2.7; $P=0.020 ; 95 \%$ CI 1.2, 6.0) of babies with LBW as compared with those who did not experience any sickness.

Table 6 presents a logistic regression analysis of the predictors of PTB. Maternal education status, LPI, HHS and maternal morbidity were significantly associated with delivering PTB. Pregnant adolescents with no formal education presented increased odds (AOR 9.0; $P=0.004 ; 95 \%$ CI 2.1, $39 \cdot 8$ ) of having PTB compared with those with formal education. Adolescents who suffered hunger had increased odds (OR 2.9; $P=0.010 ; 95 \%$ CI 1.3, 6.5) for PTB, compared with those who were not hungry. Poorer pregnant adolescents presented increased odds (OR 2.5; $P=0.014 ; 95 \%$ CI 1.2, $5.3)$ of delivering preterm babies than those who were not poor. Experiencing maternal morbidity increased the odds (AOR 3.0; $P=0 \cdot 011 ; 95 \%$ CI $1 \cdot 3,7 \cdot 0$ ) of having PTB as compared with those who did not experience any form of morbidity.

\section{Discussion}

The present study identified predictors of adverse birth outcomes among pregnant adolescents in Ghana. The findings indicated that the use of ITN, maternal education status, hunger (HHS), poverty (LPI) and maternal morbidity were the main determinants of adverse birth outcomes (LBW and PTB) among pregnant adolescents in Ghana. The prevalence of LBW and PTB reported in the present study was $15 \cdot 2$ and $12.5 \%$, respectively. Similarly, the LBW prevalence for Western Africa and Ghana reported in 2019 were 15.2 and $14 \cdot 2 \%$, respectively ${ }^{(49)}$. Our population of adolescents is, therefore, similar to the national average with regards to LBW and lower for PTB. Compared with other studies around 
Table 3. Comparison of birth outcomes by antenatal care interventions uptake and maternal factors

\begin{tabular}{|c|c|c|c|c|c|c|}
\hline \multirow[b]{2}{*}{ Variables } & \multicolumn{2}{|c|}{ Birth weight } & \multirow[b]{2}{*}{$P$ value } & \multicolumn{2}{|c|}{ Gestational age } & \multirow[b]{2}{*}{$P$ value } \\
\hline & LBW & NBW & & Preterm & Term & \\
\hline Nutrition Education & & & 0.499 & & & 1 \\
\hline Yes & $19(14)$ & $117(86)$ & & $19(12 \cdot 4)$ & $134(87 \cdot 6)$ & \\
\hline No & $24(17 \cdot 9)$ & $110(82 \cdot 1)$ & & $19(12 \cdot 7)$ & $131(87 \cdot 3)$ & \\
\hline Micronutrient Supplementation & & & $0 \cdot 29$ & & & 0.375 \\
\hline Yes & $29(17 \cdot 1)$ & $141(82.9)$ & & $26(14.4)$ & $158(85.9)$ & \\
\hline No & $12(12 \cdot 0)$ & $88(88.0)$ & & $12(10 \cdot 1)$ & $107(89 \cdot 9)$ & \\
\hline Tetanus Injection & & & 0.392 & & & $0 \cdot 861$ \\
\hline Yes & $27(16 \cdot 9)$ & $131(83 \cdot 1)$ & & $21(12 \cdot 1)$ & $153(87.9)$ & \\
\hline No & $16(12 \cdot 7)$ & $96(87 \cdot 3)$ & & $17(13 \cdot 2)$ & $112(86 \cdot 8)$ & \\
\hline Insecticide-Treated Net & & & 0.496 & & & 0.599 \\
\hline Yes & $15(13 \cdot 3)$ & $98(86 \cdot 7)$ & & $24(13 \cdot 6)$ & $153(86.4)$ & \\
\hline No & $26(16 \cdot 6)$ & $131(83 \cdot 4)$ & & $14(11 \cdot 1)$ & $112(88 \cdot 9)$ & \\
\hline Malaria Tablet Intake & & & 1 & & & 0.862 \\
\hline Yes & $18(15.4)$ & $99(84.6)$ & & $16(12 \cdot 9)$ & $108(87 \cdot 1)$ & \\
\hline No & $23(15 \cdot 0)$ & $130(85 \cdot 0)$ & & $22(12 \cdot 3)$ & $157(87 \cdot 7)$ & \\
\hline Daily intake of supplements & & & 0.235 & & & 0.863 \\
\hline Yes & $24(18 \cdot 2)$ & $108(81 \cdot 8)$ & & $18(12 \cdot 9)$ & $121(87 \cdot 1)$ & \\
\hline No & $17(12 \cdot 3)$ & $121(87 \cdot 7)$ & & $20(12 \cdot 2)$ & $144(87 \cdot 8)$ & \\
\hline Use of ITN & & & 0.038 & & & 0.061 \\
\hline Yes & $10(9 \cdot 3)$ & $96(90 \cdot 7)$ & & $14(11 \cdot 2)$ & $111(88 \cdot 8)$ & \\
\hline No & $31(19 \cdot 0)$ & $133(81 \cdot 0)$ & & $24(13.5)$ & $154(86 \cdot 5)$ & \\
\hline Maternal morbidity & & & 0.024 & & & 0.011 \\
\hline Yes & $13(27 \cdot 1)$ & $35(72.9)$ & & $13(24 \cdot 1)$ & $41(75.9)$ & \\
\hline No & $28(12 \cdot 6)$ & $194(87 \cdot 4)$ & & $25(10 \cdot 0)$ & $224(90 \cdot 0)$ & \\
\hline Mode of delivery & & & 0.589 & & & 0.536 \\
\hline Vaginal & $30(15 \cdot 8)$ & $160(84 \cdot 2)$ & & $21(9 \cdot 9)$ & $191(90 \cdot 1)$ & \\
\hline Caesarean & $6(20 \cdot 7)$ & $23(79 \cdot 3)$ & & $4(12 \cdot 9)$ & $27(87 \cdot 1)$ & \\
\hline
\end{tabular}

LBW, Low Birth Weight; NBW, Normal Birth Weight.

Bold values are significant at $P<0.05$.

Table 4. Comparison of birth outcomes by HHS, LPI, eating behaviour and dietary diversity

\begin{tabular}{|c|c|c|c|c|c|c|}
\hline \multirow[b]{2}{*}{ Variables } & \multicolumn{2}{|c|}{ Birth Weight } & \multirow[b]{2}{*}{$P$ value } & \multicolumn{2}{|c|}{ Gestational Age } & \multirow[b]{2}{*}{$P$ value } \\
\hline & LBW & NBW & & Preterm & Term & \\
\hline HHS & & & $0.003^{\mathrm{a}}$ & & & $0.028^{a}$ \\
\hline No Hunger & $25(12 \cdot 0)$ & $183(88)$ & & $22(10)$ & $198(90)$ & \\
\hline Moderate Hunger & $11(35.5)$ & $20(64.5)$ & & $11(24.4)$ & $34(75 \cdot 6)$ & \\
\hline Severe Hunger & $5(16 \cdot 1)$ & $26(83.9)$ & & $5(13 \cdot 2)$ & $33(86 \cdot 8)$ & \\
\hline LPI & & & $0.029^{a}$ & & & $0.006^{a}$ \\
\hline Low & $15(11 \cdot 6)$ & $114(88.4)$ & & $13(9 \cdot 6)$ & $123(90.4)$ & \\
\hline Moderate & $5(9 \cdot 8)$ & $46(90 \cdot 2)$ & & $3(5 \cdot 1)$ & $56(94.6)$ & \\
\hline High & $21(23 \cdot 3)$ & $69(76 \cdot 7)$ & & $22(20.4)$ & $265(79 \cdot 6)$ & \\
\hline Food Aversion & & & $0.995^{\mathrm{b}}$ & & & $1.000^{\mathrm{b}}$ \\
\hline Yes & $19(15 \cdot 2)$ & $106(84.8)$ & & $17(12.5)$ & $119(14 \cdot 6)$ & \\
\hline No & $22(15 \cdot 2)$ & $123(84.8)$ & & $21(12.6)$ & $146(87.4)$ & \\
\hline Food Craving & & & $0.818^{b}$ & & & $0.858^{\mathrm{b}}$ \\
\hline Yes & $27(14.8)$ & $155(85 \cdot 2)$ & & $25(12.9)$ & $169(87 \cdot 1)$ & \\
\hline No & $14(15.9)$ & $74(84.1)$ & & $13(11.9)$ & $96(88.1)$ & \\
\hline Pica Practice & & & $0.728^{\mathrm{b}}$ & & & $0.590^{\mathrm{b}}$ \\
\hline Yes & $16(16 \cdot 2)$ & $83(83.8)$ & & $12(10.7)$ & $100(89 \cdot 3)$ & \\
\hline No & $25(14.6)$ & $146(85.4)$ & & $26(13.6)$ & $165(86.4)$ & \\
\hline Minimum Dietary Diversity & & & $0.170^{\mathrm{b}}$ & & & $0.287^{b}$ \\
\hline Adequate & $13(11.4)$ & $101(88 \cdot 6)$ & & $18(13.7)$ & $113(86 \cdot 3)$ & \\
\hline Inadequate & $28(17.9)$ & $128(82 \cdot 1)$ & & $20(11.6)$ & $152(88.4)$ & \\
\hline
\end{tabular}

HHS, Household Hunger Scale; LPI, Lived Poverty Index; LBW, Low Birth Weight; NBW, Normal Birth Weight.

Bold values are significant at $P<0.05$.

${ }^{\mathrm{a}} \chi^{2}$.

b Fischer's exact test.

the world, our findings regarding LBW and PTB were higher ${ }^{(32,50)}$ but lower from other results ${ }^{(51,52)}$. Within Ghana, a study in Cape Coast reported a similar LBW prevalence of $14.3 \%$ among pregnant adolescents ${ }^{(36)}$.
Generally, access to ANC interventions among pregnant adolescents used in the study was relatively low. For example, less than half $(49.5 \%)$ of the participants reported having received nutrition education at ANC. The uptake of the 
Table 5. Predictors of low birth weight

\begin{tabular}{|c|c|c|c|c|}
\hline \multirow[b]{2}{*}{ Variable } & \multicolumn{4}{|c|}{ Low birth weight (LBW) } \\
\hline & $\begin{array}{l}\text { Unadjusted OR } \\
\qquad(95 \% \mathrm{Cl})\end{array}$ & $\begin{array}{c}P \\
\text { value }\end{array}$ & $\begin{array}{c}\text { AOR } \\
(95 \% \mathrm{Cl})\end{array}$ & $\begin{array}{c}P \\
\text { value }\end{array}$ \\
\hline \multicolumn{5}{|c|}{ Use of Insecticide Net } \\
\hline Yes & $0.4(0 \cdot 2-1 \cdot 0)$ & 0.038 & $0.4(0.2-0.8)$ & 0.013 \\
\hline No & 1 & & 1 & \\
\hline \multicolumn{5}{|c|}{ Lived Poverty Index } \\
\hline High & $2 \cdot 5(1 \cdot 2-5 \cdot 1)$ & 0.014 & $1.9(0 \cdot 8-4 \cdot 6)$ & 0.157 \\
\hline Moderate & $1.0(0.3-2 \cdot 7)$ & 0.883 & $0.8(0 \cdot 3-2 \cdot 3)$ & 0.657 \\
\hline Low & 1 & & 1 & \\
\hline \multicolumn{5}{|c|}{ Household Hunger Scale } \\
\hline $\begin{array}{l}\text { Moderate } \\
\text { hunger }\end{array}$ & $4 \cdot 0(1 \cdot 7-9 \cdot 3)$ & 0.001 & $3 \cdot 0(1 \cdot 1-8 \cdot 1)$ & 0.028 \\
\hline $\begin{array}{l}\text { Severe } \\
\text { hunger }\end{array}$ & $1 \cdot 4(0 \cdot 5-4 \cdot 0)$ & 0.521 & $1 \cdot 0(0 \cdot 3-3 \cdot 3)$ & 0.971 \\
\hline No hunger & 1 & & 1 & \\
\hline \multicolumn{5}{|c|}{ Maternal Morbidity } \\
\hline Yes & $2 \cdot 6(1 \cdot 2-5 \cdot 4)$ & 0.013 & $2 \cdot 7(1 \cdot 2-6 \cdot 0)$ & 0.020 \\
\hline No & 1 & & 1 & \\
\hline
\end{tabular}

Adjusted for Age and Community type, OR, Odds ratio; AOR, Adjusted odds ratio; $\mathrm{Cl}$ Confidence interval, statistically significant $p$ values are bolded.

Table 6. Predictors of preterm birth

\begin{tabular}{|c|c|c|c|c|}
\hline \multirow[b]{2}{*}{ Variable } & \multicolumn{4}{|c|}{ Preterm birth (PTB) } \\
\hline & $\begin{array}{l}\text { Unadjusted OR } \\
\qquad(95 \% \mathrm{Cl})\end{array}$ & $\begin{array}{c}P \\
\text { value }\end{array}$ & $\begin{array}{c}\text { AOR } \\
(95 \% \mathrm{Cl})\end{array}$ & $\begin{array}{c}P \\
\text { value }\end{array}$ \\
\hline \multicolumn{5}{|l|}{ Education } \\
\hline None & $5 \cdot 1(1 \cdot 4-18 \cdot 8)$ & 0.013 & $9 \cdot 0(2 \cdot 1-39 \cdot 8)$ & 0.004 \\
\hline JHS & $1 \cdot 1(0 \cdot 4-2 \cdot 6)$ & 0.874 & $1 \cdot 3(0 \cdot 5-3 \cdot 2)$ & 0.646 \\
\hline Primary & $0 \cdot 4(0 \cdot 1-2 \cdot 1)$ & 0.292 & $0 \cdot 4(0 \cdot 1-2 \cdot 4)$ & 0.325 \\
\hline SHS & 1 & & 1 & \\
\hline \multicolumn{5}{|c|}{ Lived Poverty Index } \\
\hline High & $2 \cdot 5(1 \cdot 2-5 \cdot 3)$ & 0.014 & $2 \cdot 3(0 \cdot 9-5 \cdot 8)$ & 0.087 \\
\hline Moderate & $0 \cdot 4(0 \cdot 2-2 \cdot 0)$ & 0.370 & $0 \cdot 4(0 \cdot 1-1 \cdot 5)$ & 0.182 \\
\hline Low & 1 & & 1 & \\
\hline \multicolumn{5}{|c|}{ Household Hunger Scale } \\
\hline $\begin{array}{l}\text { Moderate } \\
\text { hunger }\end{array}$ & $2 \cdot 9(1 \cdot 3-6 \cdot 5)$ & 0.01 & $2 \cdot 3(0 \cdot 9-6 \cdot 2)$ & 0.092 \\
\hline $\begin{array}{l}\text { Severe } \\
\text { hunger }\end{array}$ & $1 \cdot 4(0.5-3 \cdot 8)$ & 0.558 & $0 \cdot 8(0 \cdot 2-2 \cdot 9)$ & 0.772 \\
\hline No hunger & 1 & & 1 & \\
\hline \multicolumn{5}{|c|}{ Maternal Morbidity } \\
\hline Yes & $2 \cdot 8(1 \cdot 3-6 \cdot 0)$ & 0.006 & $3 \cdot 0(1 \cdot 3-7 \cdot 0)$ & 0.011 \\
\hline No & 1 & & 1 & \\
\hline
\end{tabular}

Adjusted for Age and Community type, OR, Odds ratio; AOR, Adjusted odds ratio; $\mathrm{Cl}$, Confidence interval, statistically significant $p$ values are bolded.

interventions by adolescents was also low. Four in ten participants did not take their micronutrient supplements, nor used the ITNs, and a few took their malaria tablets $(40 \cdot 4 \%)$. The low compliance may negate the benefits of attending ANC, as the mere attendance to ANC sessions is not the fundamental objective. This calls for programmes, including pregnant women's education in general, on the benefits of complying with these interventions. The low compliance may also be due to the fewer number of ANC sessions attended. Pregnant adolescents are likely to delay pregnancy disclosure due to uncertainty and vulnerability, especially during the first trimester, lack of funds and stigmatisation ${ }^{(30)}$. Compared with their adult counterparts, pregnant adolescents are likely to access ANC services later during pregnancy ${ }^{(53,54)}$.
Among all the ANC interventions and compliance assessed, adolescent girls from the urban areas were better off than their rural counterparts. More urban pregnant adolescents received nutrition education $(0 \cdot 022)$, tetanus injection $(0 \cdot 021)$ and had access to ITNs (although more rural girls actually used the ITNs) compared with their rural folks. Tanya and Goldenberg ${ }^{(17)}$ identified the inability to pay for services, lack of transport and prior negative experiences as barriers to healthcare services among rural women. For the present study, we think low socioeconomic status and less access to health care in rural areas are possible reasons for the lower uptake of interventions by rural girls.

The consistent and appropriate use of ITN has been one of the efficient means in preventing malaria infection by limiting the contact and bite of mosquitoes ${ }^{(55)}$. Since 2000, mass and continuous distribution networks have substantially increased access to ITNs in Ghana ${ }^{(56)}$. Notwithstanding these achievements, a significant gap exists between ITN access and usage. In our study, $58 \%$ of the pregnant adolescents reported having received ITN, while $41 \%$ were using them. Low use of ITN has been reported and associated with discomfort from the smell of chemical used, heat and difficulty installing the net ${ }^{(57)}$. Younger women (under 19 years of age) and women with less education or less hurdles to ITN usage include low awareness of ITNs by women, household or cultural constraints such as low social status or economic dependence, age, marital status, education, employment status, knowledge of malaria/ITN and IPTp reception ${ }^{(58)}$. However, our study reported a $10 \%$ less LBW deliveries in ITN non-users than among ITN users (9.3\% v. $19 \% ; P=0.037)$. This disparity may be related to the possibility that despite these adolescents receiving antenatal interventions, they were at a higher risk of other relating factors such as maternal underweight, hunger, poverty directly associated with LBW compared with those who did not receive antenatal interventions, and these factors may have confounding effects on their birth outcomes rather than the antenatal interventions.

Plasmodium falciparum infection during pregnancy is a prominent cause of LBW. In our findings, the use of ITN was significantly associated with LBW; those who used the ITN presented reduced odds of delivering babies with LBW. Similar to this result, other studies ${ }^{(59-61)}$ have reported the association of ITN use with birth outcomes. For instance, a significant reduction in the risk of LBW in healthy malaria transmission regions has been associated with the use of antimalarial intermittent pregnancy prevention (IPTp) and ITN during pregnancy ${ }^{(59)}$. A higher reduction rate of LBW $(23 \%)$ and fetal loss $(33 \%)$ with the use of ITN has also been reported among women of low gravidity in Africa ${ }^{(60)}$. Kubi Appiah and others also reported increased odds of normal birth weight deliveries (AOR 2.17;95\% CI 0.03, 0.92) to be associated with women who received a long-lasting ITN from ANC clinics ${ }^{(61)}$.

The study predicted that among all socio-demographic factors used, the education status of the mother was significantly associated with PTB. Among pregnant adolescents who had no formal education, $40 \%$ delivered PTB babies. This suggests that maternal education level affects birth outcomes. Access to education can expose an individual to information 
healthier lifestyles during pregnancy, preventing adverse effects. Similar results of low maternal education level associated with adverse birth outcomes were reported in Quebec, Canada ${ }^{(62)}$, and in two meta-analysis ${ }^{(63,64)}$. We understand the correlation between maternal education and maternofetal outcomes by the more likely disposition of women of higher education having a greater understanding of prenatal care, early initiation of prenatal care, more ANC consultations as prescribed and higher socioeconomic status ${ }^{(64,65)}$. Contrary to our findings, maternal education among Mexican American had little effect on birth outcomes ${ }^{(66)}$. They observed that the Mexican American presented the least infant mortality rate as compared with the other populations used ${ }^{(66)}$.

Hunger and poverty presented significant associations with adverse birth outcomes. Hunger led to an increased likelihood of having adverse birth outcomes in the present study. Pregnant adolescents who experienced hunger and were poorer using HHS and LPI, respectively, presented significantly higher odds of giving birth to LBW and preterm babies than those who did not experience any hunger or deprivation. The possible reason for more adverse birth outcomes among pregnant adolescents who experienced poverty and hunger is inadequate maternal dietary intake and low nutritional quality diet, as these have been related to adverse birth outcomes ${ }^{(11)}$. During pregnancy, the intake of adequate dietary diversity diet offers the required micronutrients that are important for the growth and development of the fetus. However, our findings did not present a significant relationship between dietary diversity and adverse birth outcomes though a greater proportion of LBW babies were found among adolescents with inadequate dietary diversity as compared with those with adequate dietary diversity. Contrary to our findings, Quansah and Boateng ${ }^{(67)}$ found inadequate dietary diversity to be associated with higher odds of LBW babies. Maternal nutritional status can have an impact on fetal development. The proper growth and development of the fetus requires the consumption of food items from the various food groups in order to prevent deficiencies of essential micronutrients. Thus, adequate dietary intake is critical in preventing adverse birth outcomes. In developing countries, poor nutritional status is a determining factor of LBW due to low nutrient flow to the developing fetus ${ }^{(68)}$. Experiencing hunger during pregnancy can be devastating and can be subsequently linked to poor nutritional status as the pregnancy period requires a significant increase in macronutrients and adequate micronutrients. According to a study on fetal response to satiation and hunger, $74 \%$ of pregnant women experienced an increase in fetal activity when they were hungry. The paper further stated that women with increased fetal activity gave birth to smaller babies ${ }^{(69)}$. Studies have related poverty to adverse birth outcomes such as LBW, PTB and fetal loss ${ }^{(17,70)}$. Tanya ${ }^{(17)}$ further asserted that the poor might have less access to healthcare systems and maternal care during emergencies. A high risk of malnutrition and subsequent adverse effect on babies leading to nutritional and developmental deficiencies have also been related to poverty ${ }^{(71)}$.

Maternal morbidity was significantly associated with birth outcomes in the present study. The odds of having LBW and PTB among those who got ill during pregnancy were thrice more than those who did not experience any illness. This implies that the health of the mother can be a contributory factor to birth outcomes. According to Tinker ${ }^{(20)}$, maternal malnourishment and insufficient maternal care are high risks for the development of diseases and death during delivery. This is expected since maternal illness can affect fetal growth and development. Some of the conditions that these pregnant adolescents went through during pregnancy were pregnancy induced hypertension, antepartum haemorrhage and prolonged labour. These findings seem to be consistent with earlier research which reported that participants who experienced morbidity had about five to seven times increased risk of adverse birth outcomes ${ }^{(21,72,73)}$.

Generally, the effect of geographical location, environmental conditions, socio-cultural practices, eating behaviour and maternal factors on birth outcomes have been mixed. For instance, in the present study, LBW was more prevalent among rural girls $(17 \%)$ than the urban participants $(13.7 \%)$, similar to a study conducted in Malaysia ${ }^{(74)}$, but contrary to other studies in Ghana ${ }^{(36,75,76)}$, which reported a higher prevalence of LBW among urban than rural participants. A higher prevalence of LBW is expected in rural areas due to poorer socioeconomic conditions, food insecurity, low nutrition knowledge and limited access to health care ${ }^{(77-79)}$. In the present study, the prevalence of caesarean delivery, $11.8 \%$, was similar to studies conducted in Ghana ${ }^{(76)}$ but lower than two studies conducted in Bangladesh ${ }^{(80)}$ and Pakistan ${ }^{(70)}$, which recorded 23.9 and $26.4 \%$, respectively. A greater proportion of caesarean deliveries in this study $(16.4 \%)$ were from the urban centres. This may seem to be due to urbanisation ${ }^{(81)}$ and access to qualified personnel to carry out the section. Hasan $^{(80)}$ also presented similar findings where the rate of caesarean deliveries was relatively high in urban women than in rural women. Caesarean sections are less accessible among poor or low-income countries ${ }^{(82)}$. Generally, about $21 \%$ of those who delivered through caesarean section gave birth to LBW babies. Literature suggests that caesarean sections are usually performed as emergencies without adequate preparations and can lead to a challenge in the newborn's proper initial development ${ }^{(83)}$.

The overall nutritional status such as weight gain, health, parity, previous birth outcomes and biochemical status of the mother during pregnancy may affect birth outcomes. Some studies have presented an increased odds of obstetric, placental and medical complications with increasing parity ${ }^{(84-86)}$. Furthermore, poor socioeconomic status and its consequences, such as less access to health facilities and low levels of education have been associated with adverse birth outcomes $^{(87,88)}$. Moreover low birth weight have been associated with pregnant women with chronic anaemia, diabetes and hypertension ${ }^{(84,89)}$

In summary, this study explored the predictors of adverse birth outcomes among pregnant adolescents, and among many factors found that hunger, poverty and lack of formal education predicted preterm births. Pregnant girls with no formal education had nine times increased odds for PTB compared with those with at least secondary education. Girls 
with high LPI had two and a half increased odds for PTB compared with those with no poverty, and those who experienced moderate hunger were about two and a half times increased odds for PTB compared with those with no hunger. Finally, those who were ill during pregnancy were thrice increased odds for PTB compared those who were not ill. For LBW, the significant predictors were similarly hunger, poverty and maternal morbity. The levels of the odds recorded for the predictors of $\mathrm{LBW}$ were simlar to that of the PTB. On the other hand, the use of ITN reduced the odds for LBW deliveries by about $60 \%$.

\section{Limitation}

The study had a significant dropout as less than $75 \%$ of baseline participants were followed up for the birth outcomes study. Future studies should be designed to reduce this, either by increasing the sample size or improve measures for tracking participants.

\section{Conclusion}

The risk of adverse birth outcomes observed among the pregnant adolescents were associated with ANC compliance and maternal socioeconomic factors. Strengthening antenatal services, promoting girl education, livelihood support for pregnant girls, and interventions to improve socio-economic status and to address hunger and poverty are broad and targeted interventions to both reduce the incidence of adolescent pregnancies, and reduce the risk for adverse birth outcomes among those who still become pregnant. Focused care to encourage attendance to ANC and improve compliance to ANC interventions by pregnant adolescents is strongly recommended. Food security and hunger should be evaluated during antenatal services to identify and address the vulnerable.

\section{Acknowledgements}

The authors thank the Health Directors at the health centres where the research was done, and most of all the pregnant adolescents who participated in the study.

The Nestle Foundation provided funding for this research. The funder had no role in the design, analysis or writing of this article.

Formulating the research questions: R. A. A., C. A., L. A. G., H. E. L., W. A. and A. K. E.; designing the study: R. A. A., C. A., H. E. L., L. N., W. A. and A. K. E.; carrying it out: R. A. A., C. A., L. A. G., L. N., H. E. L., W. A. and O. A.-B.; analysing the data: R. A. A., L. A. G., O. A.-B. and C. A.; writing the article: R. A. A., L. A. G., C. A., H. E. L., W. A. and A. K. E.

The authors declare that they have no conflicts of interest.

\section{References}

1. EFCNI (2018) Why the First 1000 Days of Life Matter, pp. 1-4. https://www.efcni.org/wp-content/uploads/2018/05/2018_04_23_ EFCNI_1000Tage_Factsheet_web.pdf.
2. Wadhwa DP, Glynn L, Hobel CJ, et al. (2002) Behavioral perinatology: biobehavioral processes in human fetal development. Regul Pept 108, 149-157.

3. World Health Organization (2014) WHO Global Nutrition Targets 2025: Anaemia Policy Brief. Geneva: WHO.

4. Risnes KR, Vatten LJ, Baker JL, et al. (2011) Birthweight and mortality in adulthood: a systematic review and metaanalysis. Int $J$ Epidemiol 40, 647-661.

5. Kim D \& Saada A (2013) The social determinants of infant mortality and birth outcomes in western developed nations: a crosscountry systematic review. Int J Environ Res Public Health 10, 2296-2335.

6. Reinebrant HE, Leisher SH, Coory M, et al. (2018) Making stillbirths visible: a systematic review of globally reported causes of stillbirth. BJOG 125, 212-224.

7. Brown H, Speechley K, Macnab J, et al. (2014) Biological determinants of spontaneous late preterm and early term birth: a retrospective cohort study. BJOG 122, 491-499, https://doi.org/10. 1111/1471-0528.13191.

8. Kramer MS, Séguin L, Lydon J, et al. (2000) Socio-economic disparities in pregnancy outcome: why do the poor fare so poorly? Paediatr Perinat Epidemiol 14, 194-210.

9. Laraia BA, Siega-Riz AM \& Gundersen C (2010) Household food insecurity is associated with self-reported pregravid weight status, gestational weight gain, and pregnancy complications. J Am Diet Assoc 110, 692-701.

10. Maddah M, Karandish M, Mohammadpour-Ahranjani B, et al. (2005) Social factors and pregnancy weight gain in relation to infant birth weight: a study in public health centers in Rasht, Iran. Eur J Clin Nutr 59, 1208-1212.

11. Abu-Saad K \& Fraser D (2010) Maternal nutrition and birth outcomes. Epidemiol Rev 32, 5-25.

12. Lumey LH \& Stein AD (1997) Offspring birth weights after maternal intrauterine undernutrition: a comparison within sibships. $A m \mathrm{~J}$ Epidemiol 146, 810-819.

13. Selling KE, Carstensen J, Finnström O, et al. (2006) Intergenerational effects of preterm birth and reduced intrauterine growth: a population-based study of Swedish mother-offspring pairs. BJOG 113, 430-440.

14. Sintayehu H \& Bedasa W (2019)Dietary diversity and associated factors among pregnant women attending antenatal care at public health facilities in Bale Zone, Southeast Ethiopia. Nutrition and Dietary Supplements 11, 1-8, https://doi.org/10.2147/NDS.S179265.

15. Abubakari A \& Jahn A (2016) Maternal dietary patterns and practices and birth weight in Northern Ghana. PLOS ONE 11, e0162285.

16. Gyimah LA, Annan RA, Apprey C, et al. (2021) Dietary diversity and its correlates among pregnant adolescent girls in Ghana. PLOS ONE 16, e0247979.

17. Tanya Nagahawatte N \& Goldenberg RL (2008) Poverty, maternal health, and adverse pregnancy outcomes. Ann N Y Acad Sci 1136, $80-85$.

18. Aga Khan University \& UNICEF (2001) Pakistan National Nutrition Survey (NNS). Retrieved from Islamabad: http://pakresponse.info/LinkClick.aspx?leticket $=$ Ao $4 \mathrm{~s}-$ rwdFVI\%3D\&tabid $=$ $117 \&$ mid $=752$ (accessed 28 June 2020)

19. Kassebaum NJ, Bertozzi-Villa A, Coggeshall MS, et al. (2014) Global, regional, and national levels and causes of maternal mortality during 1990-2013: a systematic analysis for the Global Burden of Disease Study 2013. Lancet 384, 980-1004.

20. Tinker A (2000) Women's Health: The Unfinished Agenda. International Journal of Gynecology \& Obstetrics 2000(70), 149-158.

21. Yeshialem E, Alemnew N, Abera M, et al. (2017) Determinants of adverse pregnancy outcomes among mothers who gave birth from Jan 1-Dec 31/2015 in Jimma University specialized hospital, case control study, 2016 study area and period. Med Clin Rev. 3(22), $1-10$.

22. Nisar N \& White F (2003) Factors affecting utilization of antenatal care among reproductive age group women (15-49 years) in an urban squatter settlement of Karachi. J Pak Med Assoc 53, 47-53. 
23. World Health Organization (WHO) (2019) World Health Statistics 2019: Monitoring Health for the SDGS, Sustainable Development Goals [Internet]. https://apps.who.int/iris/bitstream/handle/10665/324835/ 9789241565707-eng.pdf (cited June 2021).

24. Kuhnt J \& Vollmer S (2017) Antenatal care services and its implications for vital and health outcomes of children: evidence from 193 surveys in 69 low-income and middle-income countries. BMJ Open 7, e017122.

25. Afulani AP (2016) Determinants of stillbirths in Ghana: does quality of antenatal care matter? BMC Pregnancy Childbirth 16, 132.

26. Bullough C, Meda N, Makowiecka K, et al. (2005) Current strategies for the reduction of maternal mortality. BJOG 112, 1180-1188.

27. Magadi M, Madise N \& Diamond I (2001) Factors associated with unfavourable birth outcomes in Kenya. J Biosoc Sci 33, 199-225.

28. WHO \& UNICEF (2003) Antenatal Care in Developing Countries: Promises, Acbievements and Missed Opportunities - An Analysis of Trends, Levels and Differentials, 1990-2001. World Health Organization.

29. Baffour-Awuah A, Mwini-Nyaledzigbor PP \& Richter S (2015) Enhancing focused antenatal care in Ghana: an exploration into perceptions of practicing midwives. Int J Africa Nurs Sci 2, 59-64.

30. Pell C, Meñaca A, Were F, et al. (2013) Factors affecting antenatal care attendance: results from qualitative studies in Ghana, Kenya and Malawi. PLOS ONE 8, e53747.

31. Alam N (2000) Teenage motherhood and infant mortality in Bangladesh: maternal age-dependent effect of parity one. J Biosoc Sci 32, 229-236.

32. Ganchimeg T \& Ota E (2014) Pregnancy and childbirth outcomes among adolescent mothers: a World Health Organization multicountry study. BJOG 121(Suppl. 1), 40-48.

33. Gibbs C, Wendt A, Peters S, et al. (2012) The impact of early age at first childbirth on maternal and infant health. Paediatr Perinat Epidemiol 26, 259-284.

34. Sharma V, Katz J, Mullany LC, et al. (2008) Young maternal age and the risk of neonatal mortality in rural Nepal. Arch Paediatr Adolesc Med 162, 828-835.

35. Weng Y-H, Yang C-Y \& Chiu Y-W (2014) Risk assessment of adverse birth outcomes in relation to maternal age. PLOS ONE $\mathbf{9}$, e114843.

36. Afriyie J, Bedu-Addo K, Asiamah E, et al. (2016) Low birth weight among adolescents at cape coast metropolitan hospital of Ghana. Int J Reprod Contraception, Obstet Gynecol 5, 4242-4247.

37. Cooper LG, Leland NL \& Alexander G (1995) Effect of maternal age on birth outcomes among young adolescents. Soc Biol 42, 22-35.

38. WHO (2018) Guideline: Implementing Effective Actions for Improving Adolescent Nutrition [Internet]. WHO. Licence: CC BY-NC-SA 3.0 IGO. http://apps.who.int/iris/bitstream/handle/10665/260297/ 9789241513708-eng.pdf;jsessionid=19D1CBFA434795BA1645CC 009FFE99A4? sequence $=1$.

39. Ghana Statistical Service (GSS), Ghana Health Service (GHS) \& ICF (2018) Ghana Maternal Health Survey 2017: Key Indicators Report [Internet]. Accra G. Ghana Maternal Health Survey. http://www. statsghana.gov.gh/docfiles/PR95.pdf\%00Ahttps://dhsprogram.com/ pubs/pdf/PR95/PR95.pdf.

40. Asundep NN, Carson AP, Archer C, et al. (2013) Determinants of access to antenatal care and birth outcomes in Kumasi, Ghana. J Epidemiol Glob Health 3, 279-288.

41. Yatich JN, Yi J, Agbenyega T, et al. (2009) Malaria and intestinal helminth co-infection among pregnant women in Ghana: prevalence and risk factors. Am J Trop Med Hyg 80, 896-901.

42. Ayensu J, Edusei A, Oduro I, et al. (2017) Status of some antioxidant micronutrient and pregnancy outcomes in Ghanaian adolescents attending antenatal clinic in urban (Suntreso) and rural (Mampong) hospitals. Eur J Nutr Food Saf 7, 120-127.

43. MoGCSP (2017) Adolescent Pregnancy Strategic Plan, pp. 1-5. https:// ghana.unfpa.org/sites/default/files/pub-pdf/Adolescent Pregnancy Strategic Plan 2018.pdf.

44. Ghana Statistical Service (2014) 2010 Population and Housing Census: District Analytic Report, Kumasi Metropolitan [Internet]. https://www. google.com/search?client=firefox-b-d\&q=Ghana+Statistical+Service.
$+2010+$ Population +and +housing + census $\% 3 \mathrm{~A}+$ District+analytic + report $\% 2 \mathrm{C}+$ Kumasi+Metropolitan. +2014 . + Available + online $\% 3 \mathrm{~A}+$ http $\% 3 \mathrm{~A} \% 2 \mathrm{~F} \% 2 \mathrm{Fwww} 2$. statsghana.gov.gh\%2Fdocfiles $\% 2 \mathrm{~F} 2010$ District_R (cited May 2021).

45. Charan J \& Biswas T (2013) How to calculate sample size for different study designs in medical research? Indian J Psychol Med 35, 121-126.

46. Ballard T, Coates J, Swindale A, et al. (2011) Household hunger scale: indicator definition and measurement guide. Nutrition, 6-13.

47. Mattes R, Dulani B, Gyimah-Boadi E (2016) Africa's growth dividend? Lived poverty drops across much of the continent.

48. Lawn JE, Gravett MG, Nunes TM, et al. (2010) Global report on preterm birth and stillbirth (1 of 7): definitions, description of the burden and opportunities to improve data. BMC Pregnancy Childbirth 10, S1.

49. UNICEF \& WHO (2019) Low birthweight estimates.

50. Bihoun B, Zango SH, Coulibaly MT, et al. (2017) Low birth weight and prematurity in teenage mothers in rural areas of Burkina Faso. J Pregnancy Child Health 04, 344.

51. Mombo-Ngoma G, Mackanga JR, González R, et al. (2016) Young adolescent girls are at high risk for adverse pregnancy outcomes in Sub-Saharan Africa: an observational multicountry study. BMJ Open 6, e011783.

52. Usynina A, Postoev V, Odland J, et al. (2018) Adverse pregnancy outcomes among adolescents in northwest Russia: a population registry-based study. Int J Environ Res Public Health 15, 261.

53. Blondel B, Dutilh P, Delour M, et al. (1993) Poor antenatal care and pregnancy outcome. Eur J Obstet Gynecol Reprod Biol 50, 191-196.

54. Kirbas A, Gulerman HC \& Daglar K (2016) Pregnancy in adolescence: is it an obstetrical risk? J Pediatr Adolesc Gynecol 29, 367-371.

55. Yirsaw AN, Gebremariam RB, Getnet WA, et al. (2021) Insecticide-treated net utilization and associated factors among pregnant women and under-five children in East Belessa district, northwest Ethiopia: using the health belief model. Malar J 20, 130.

56. Ahorlu CS, Adongo P, Koenker H, et al. (2019) Understanding the gap between access and use: a qualitative study on barriers and facilitators to insecticide-treated net use in Ghana. Malar J 18, 417.

57. Manu G, Boamah-Kaali EA, Febir LG, et al. (2017) Low utilization of insecticide-treated bed net among pregnant women in the middle belt of Ghana. Malar Res Treat 2017, 7481210.

58. Hill J, Hoyt J, van Eijk AM, et al. (2013) Factors affecting the delivery, access, and use of interventions to prevent malaria in pregnancy in Sub-Saharan Africa: a systematic review and meta-analysis. PLoS Med 10, e1001488.

59. Eisele TP, Larsen DA, Anglewicz PA, et al. (2012) Malaria prevention in pregnancy, birthweight, and neonatal mortality: a metaanalysis of 32 national cross-sectional datasets in Africa. Lancet Infect Dis 12, 942-949.

60. Gamble C, Ekwaru PJ, Garner P, et al. (2007) Insecticide-treated nets for the prevention of malaria in pregnancy: a systematic review of randomised controlled trials. PLoS Med 4, e107.

61. Appiah PK, Bukari M, Yiri-Erong SN, et al. (2020) Antenatal care attendance and factors influenced birth weight of babies born between June 2017 and May 2018 in the Wa East District, Ghana. Int J Reprod Med 2020, 10.

62. Luo ZC, Wilkins R \& Kramer MS (2006) Effect of neighbourhood income and maternal education on birth outcomes: a populationbased study. CMAJ 174, 1415-1420.

63. Ruiz M, Goldblatt P, Morrison J, et al. (2015) Mother's education and the risk of preterm and small for gestational age birth: a DRIVERS meta-analysis of 12 European cohorts. J Epidemiol Community Health 69, 826-833.

64. Silvestrin S, Da Silva CH, Hirakata VN, et al. (2013) Maternal education level and low birth weight: a meta-analysis. J Pediatr 89, 339-345.

65. Haider BA \& Bhutta ZA (2017) Multiple-micronutrient supplementation for women during pregnancy. Cochrane Database Syst Rev 4, CD004905. 
66. Gage TB, Fang F, O’Neill E, et al. (2013) Maternal education, birth weight, and infant mortality in the United States. Demography 50, 615-635.

67. Quansah DY \& Boateng D (2020) Maternal dietary diversity and pattern during pregnancy is associated with low infant birth weight in the Cape Coast Metropolitan Hospital, Ghana: a hospital based cross-sectional study. Heliyon 6, e03923.

68. Biesalski HK, Black RE \& Koletzko B (2016) Hidden Hunger: Malnutrition and the First 1,000 Days of Life: Causes, Consequences and Solutions, vol. 115. New York: Karger Medical and Scientific Publishers.

69. Bradford B \& Maude R (2014) Fetal response to maternal hunger and satiation - novel finding from a qualitative descriptive study of maternal perception of fetal movements. BMC Pregnancy Childbirth 14, 288.

70. Aftab S, Ainuddin J, Kazi S, et al. (2012) Effects of poverty on pregnant women. Pak J Med Res 51(1), 5-9.

71. Black RE, Allen LH, Bhutta ZA, et al. (2008) Maternal and child undernutrition: global and regional exposures and health consequences. Lancet 371, 243-260.

72. Chaibva BV (2014) Determinants of adverse pregnant outcomes in Mutare district clinics, Manicaland Province, Zimbabwe (Doctoral dissertation, University of Pretoria). http://hdl.handle.net/2263/46131.

73. Sadiq Abubakar A, Poggensee G, Nguku P, et al. (2016) Factors associated with adverse pregnancy outcomes and perceptions of risk factors among reproductive age women in Soba LGA, Kaduna state 2013. Pan Afr Med J 25, 1-8.

74. Kaur S, Ng CM, Badon SE, et al. (2019) Risk factors for low birth weight among rural and urban Malaysian women. BMC Public Health 19(Suppl. 4), 539, https://doi.org/10.1186/s12889-019-6864-4.

75. Abubakari A, Kynast-Wolf G \& Jahn A (2015) Prevalence of abnormal birth weight and related factors in northern region, Ghana. BMC Pregnancy Cbildbirth 15.

76. Tampah-Naah A, Anzagra L \& Yendaw E (2016) Factors correlate with low birth weight in Ghana. BrJ Med Med Res 16, 1-8.
77. Payghan BS, Kadam SS \& Reddy RM (2014) A comparative study of nutritional awareness among urban-rural pregnant mothers. Res Rev J Med Heal Sci 3, 95-99.

78. Suliga E (2015) Nutritional behaviours of pregnant women in rural and urban environments. Ann Agric Environ Med 22(3), 513-517.

79. Tran TK, Nguyen CT, Nguyen HD, et al. (2011) Urban-rural disparities in antenatal care utilization: a study of two cohorts of pregnant women in Vietnam. BMC Health Serv Res 11, 120.

80. Hasan F, Alam MM \& Hossain MG (2019) Associated factors and their individual contributions to caesarean delivery among married women in Bangladesh: analysis of Bangladesh demographic and health survey data. BMC Pregnancy Childbirth 19, 433.

81. de Loenzien M, Schantz C, Luu BN, et al. (2019) Magnitude and correlates of caesarean section in urban and rural areas: a multivariate study in Vietnam. PLoS ONE 14, e0213129.

82. Ronsmans C, Holtz S \& Stanton C (2006) Socioeconomic differentials in caesarean rates in developing countries: a retrospective analysis. Lancet 368, 1516-1523.

83. The Partnership for Maternal \& Child Health (2006) Opportunities for Africa's Newborns, vol. 250.

84. Aliyu MH, Jolly PE, Ehiri JE, et al. (2005) High parity and adverse birth outcomes: exploring the maze. Birth 32, 45-59.

85. Brunner J, Melander E, Krook-Brandt M, et al. (1992) Grand multiparity as an obstetric risk factor: a prospective case-control study. Eur J Obstet Gynecol Reproduct Biol 47, 201-205.

86. Eskenazi B, Fenster L \& Jama SS (1991) A multivariate analysis of risk factors for preeclampsia. JAMA 266, 237-241.

87. Nordin NM, Fen CK, Isa S, et al. (2006) Is grandmultiparity a significant risk factor in this new millennium? Malaysian J Med Sci 13, $52-60$.

88. Ozumba BC \& Igwegbe AO (1992) The challenge of grandmultiparity in Nigerian obstetric practice. Int J Gynecol Obstet 37, 259-264.

89. Shah PS (2010) Parity and low birth weight and preterm birth: a systematic review and meta-analyses. Acta Obstet Gynecol Scand 89, 862-875. 